\title{
GROUND STATE PHASE DIAGRAM OF MIXED-STACK COMPOUNDS WITH INTERMOLECULAR ELECTRON TRANSFER
}

\author{
TADEusz LuTY
}

Institute of Physical and Theoretical Chemistry, Technical University of Wrocław Wyb. Wyspiańskiego 29, 50-370 Wrocław, Poland and

Groupe Matiere Condensée et Materiaux, URA au CNRS 804

Université de Rennes I, 35042 Rennes, Cedex, France

(Received January 12, 1995)

Dedicated to Professors Krrzysztof Pigoń, Józef W. Rohleder and Zdzisław Ruziewicz on the occasion of their 70th birthday*

The ground state energy for a chain of donor and acceptor molecules (mixed-stack architecture) is calculated within the three-state model. The model describes the intermolecular electron transfer and, in particular, stresses the role of the diagonal coupling of the electron to symmetry breaking molecular displacements and the local electric field. The modulation of the intcrmolecular Coulomb interaction is shown to have important consequences for the ground state and its dynamics. In particular, the ground state energy as a function of the displacement may show one, two or three minima with varied molecular ionicity. An analysis of the function gives a phase diagram which indicates a possibility for the coexistence of neutral (undistorted) and ionic (distorted) chains of molecules in the ground state. The function is illustrated by numerical calculations with parameters appropriate for the tetrathiafulvalene-chloranil crystal which undergoes a neutral-to-ionic phase transition induced by either temperature or pressure. The effect of the electron transfer on the lattice dynamics of the mixed-stack system is briefly considered. It is suggested that the thermodynamical phase diagram for tetrathiafulvalene-chloranil system can be understood as a result of two effects: pressure induced quantum mixing between diabatic states which determine a nature of components and temperature stimulated classical mixing of the components.

PACS numbers: $71.35 .+z, 71.38 .+\mathrm{i}$

*Submitted on invitation of the Institute of Physical and Theoretical Chemistry, Technical University of Wrocław, Wrocław, Poland. 


\section{Introduction}

Since 1981 when Torrance and co-workers [1] discovered the "neutral-to-ionic" phase transition in charge-transfer (CT) molecular crystals with mixed-stack (MS) architecture, extensive studies on the nature of the transformation have been performed. It is believed that these investigations will uncover factors responsible for electron-induced structural changes in molecular solids and allow for more applications of these materials.

Soon after the first experiments performed on the TTF-CA (tetrathiafulvalene-chloranil) crystal [2], it became clear that a temperature induced neutral-ionic, first order, phase transition is unusual. Recent experiments on TTF-CA [3] have indicated a narrow region of coexistence (disorder ?) of neutral and ionic phases as well as a change in the mechanism of the transformation under pressure. The coexistence of neutral and ionic molecules has been even more pronounced in observations of the recently discovered neutral-ionic transitions in the systems TMB-TCNQ $\left(3,3^{\prime}, 5,5^{\prime}\right.$-tetramethylbenzidine-7,7,8,8-tetracyanoquinodimethane) [4] and DMTTF-CA (dimethyltetrathiafulvalene-chloranil) [5]. There are two important features of these phase transitions: the structural change, a symmetry breaking dimerization of the MS, and a change in molecular ionicity. Although the ionicity is well understood in a qualitative sense, it is not uniquely defined quantitatively [6]. While it is not directly measurable, a totally symmetric molecular deformation may serve as its measure $[7,8]$. The changes become more continuous under pressure.

As the molecular ionicity and the lattice dimerization are coupled, it is legitimate to consider the transition as a structural one that is driven by the intermolecular electron transfer. The intramolecular and intermolecular structural changes then, in turn, enhance the electron transfer by a change in the potential difference (the diagonal coupling) and/or an increase in the CT transfer integral (off-diagonal coupling). Most theoretical models are based on a two-level Peierls-Hubbard Hamiltonian and only off-diagonal coupling is included. Important physics of the MS has been missing - the diagonal coupling of the electron transfer to the symmetry breaking dimerization via modulation of the intersite Coulomb interaction. Extensive studies on CT exciton states in MS organic crystals have shown that the modulation of the Coulomb interaction by lattice vibrations is the essence of the mechanism of strong CT exciton-phonon coupling [9]. This conclusion from the spectroscopy of CT excitons has been overlooked.

The CT exciton has been proved to be very polar. With the electron and hole on separate molecules, strong coupling to the lattice is expected and manifestations of this interaction are the broad and relatively structureless absorption spectra of MS systems. Another consequence of strong exciton-lattice coupling is relaxation of the nuclei towards a new equilibrium position which can lead at low temperatures to self-trapping of the CT exciton. In the collapsed state the molecules are envisaged as having moved closer together and/or reoriented in a way that lowers the energy of the excited state. The three-state model (neutral and two CT states) with diagonal coupling to lattice displacements that is constructed in this paper follows the above scenario towards a change in the ground state. The fact that the 
ionic-to-neutral transition in the TTF-CA crystal has been photoinduced [10] may support this line of reasoning. The diagonal coupling has been, in fact, included in the model of Horovitz and Solyom [11], but they have made a numerical analysis and did not uncover important consequences of the coupling for the ground state of MS systems. I show here that the potential function assumed by Nagaosa [12] in his phenomenological theory of the neutral-ionic phase transition can be derived and seen to be a consequence of the postulated diagonal electron transfer-phonon coupling.

The aim of the paper is to stress and uncover consequences of the diagonal electron transfer-molecular displacement coupling for the ground state of MS compounds. First, the three state model Hamiltonian will be constructed and analyzed, then the ground state energy function will be used to construct a phase diagram. This will be done by numerical illustration of an exact solution and in an approximate way by perturbation expansion.

\section{System definition and ground state energy}

The system is defined as a centrosymmetric array of alternating molecules (MS): electron donors (D) and acceptors (A), $a$ distance apart. We are interested in the process:

$$
\ldots \text { ADADADA } \ldots \Rightarrow \begin{aligned}
& \ldots \mathrm{AD}\left(\mathrm{A}^{-} \mathrm{D}^{+}\right)_{n} \mathrm{ADA} \ldots \\
& \ldots \mathrm{ADA}\left(\mathrm{D}^{+} \mathrm{A}^{-}\right)_{n} \mathrm{DA} \ldots
\end{aligned}
$$

e.g., the formation, in general, of $n$ pairs of ionized molecules, accompanied by a structural change.

The states corresponding to the electronic structure of neutral and ionized molecules are denoted as $\langle N|$ and $\left\langle I_{n}+\right|,\left\langle I_{n}-\right|$, respectively. The ionic states are characterized by opposite polarization. The configurational space in which the electron transfer occurs is defined by intramolecular $(Q)$ and intermolecular $(q)$ translational normal coordinates. For the electron transfer process, characterized by a dipole moment, $\mu$, dielectric properties of the crystal are important and they will be described by an electric field variable, $\varepsilon(p)$, expressed in terms of normal coordinates of molecular excitons $(p)$. Rotational displacements of molecules will be neglected, although as they preserve center of inversion symmetry they modify the electronic energy in the same way as intramolecular, totally symmetric coordinates. I shall consider ground state properties of MS except magnetic ones and spin degrees of freedom will be neglected. This simplification has been often used in studies of these systems where charge bistability is of main concern rather than spin bistability. It allows to keep the model to a minimum number of parameters and "transparent" enough to show the effect of diagonal electron-phonon coupling on ground state properties. In previous studies [13] of the ground state phase diagram, where off-diagonal coupling has been considered, the spin degrees of freedom have been neglected as well.

The problem of the $n$-fold electron transfer in the ...DA...chain is considered as resulting from the electronic interaction between states $\langle N|$ and $\left\langle I_{n}+\right|$, 
$\left\langle I_{n}-\right|$. The ionic states are coupled to the neutral one through an electron transfer integral, $\left\langle N|H| I_{n}+(-)\right\rangle=-t(n)$. The total Hamiltonian, $H$, is split into an electron transfer part, $H_{\mathrm{e}}$, and $H_{\mathrm{b}}$, which describes the configurational space. As for the electronic part, the $n$-fold transfer of an electron in the ...DA...chain costs energy,

$$
H_{\mathrm{e}}=\delta_{n}^{0}+g Q+\sigma(\beta q+\mu \varepsilon)-t(n) .
$$

$\delta_{n}^{0}$ denotes energy (per pair) of $n$-fold charge transfer in the absence of any coupling and may be expressed as

$$
\delta_{n}^{0}=I-A-\alpha(n) J .
$$

$I$ is ionization energy of $\mathrm{D}$, and $A$ is the electron affinity of A molecule. The last term in Eq. (2) describes intersite Coulomb interaction as a product of the Madelung constant [14],

$$
\alpha(n)=-\frac{1}{n} \sum_{k=1}^{2 n} \sum_{l>k}^{2 n}(-1)^{(k+l)}(l-k)^{-1}
$$

and $J \equiv\left\langle e^{2} / a\right\rangle$ which is Coulomb integral for nearest $\mathrm{D}$ and $\mathrm{A}$ molecules. On writing the on-site potential for $n$-fold electron transfer as in Eq. (2), I have followed the arguments of Ref. [14]. It is an approximation and would be exact only for $n=1$. This corresponds to the trimer model well-elaborated by Girlando and Painelli [15]. They have also critically checked how far one can go with the model when discussing an MS system, and indicated that the trimer model is not a bad approximation.

The second term in Eq. (1) represents a modification of ionization energy and electron affinity due to totally symmetric, intramolecular vibrations. The coupling has been elaborated in detail previously $[15,16]$. In the third term of Eq. (1), $\sigma=(+,-)$ denotes polarization of the ionic states in the coupling to the electric field and to "ungerade" lattice modes (translational). The coupling constant, $\beta$, measures modulation of the Coulomb integral, $J$, by molecular displacements in the pattern represented by the normal coordinate, $q$. For clarity we keep all quantities as scalars. The fact that the translational displacement of a molecule in the MS modifies the on-site potential in the same way as an electric field is the essence of the model. It will also bring extra strength to the effective coupling constant as will be shown latter.

The configurational space in $Q, q$ and $\varepsilon$ coordinates, which forms a bath for the electron transfer, is most simply characterized by three harmonic oscillators,

$$
H_{\mathrm{b}}=\frac{1}{2} \Omega^{2} Q^{2}+\frac{1}{2} \omega^{2} q^{2}-k q \varepsilon+\frac{1}{2} \chi^{-1} \varepsilon^{2} .
$$

$\chi$ has the sense of electronic susceptibility and $k$ couples intermolecular vibrations to the electric field since both $q$ and $\varepsilon$ are symmetry-breaking variables (the effect being represented in "shell models" of lattice dynamics). The totally symmetric, intramolecular coordinate, $Q$, does not couple directly to $q$ and $\varepsilon$ variables. Higher order couplings, like $Q q^{2}$, may be introduced and they lead to the molecular compressibility concept [8]. The normal mode spectrum of the system is such that the 
exciton and intramolecular frequencies are larger than intermolecular ones. Therefore, the variables, $\varepsilon$ and $Q$, can follow the changes in intermolecular distance, $q$, and can be eliminated from the total Hamiltonian, $H_{\mathrm{e}}+H_{\mathrm{b}}$, with the result

$$
H=\frac{1}{2}\left(\omega^{2}-k^{2} \chi\right) q^{2}+\delta(n)+\sigma(\ddot{\beta}+\mu k \chi) q-t(n),
$$

where

$$
\delta(n)=\delta_{n}^{0}-\frac{1}{2} \mu^{2} \chi-\frac{1}{2} g^{2} \Omega^{-2}
$$

is the energy of $n$-fold electron transfer as it is renormalized due to coupling with the high-frequency electric field of excitons and intramolecular deformation. The second term in Eq. (5) stands for the polarization energy correction (a "solvent effect") and the last term is the small polaron binding energy, indicating how much energy the system has gained due to totally symmetric deformation of the molecules. Both contributions decrease the on-site potential which drives the intermolecular electron transfer.

Let us observe, from Eq. (4), that the frequency of the lattice mode responsible for intermolecular deformation is renormalized in the same way as in the dielectric theory of molecular crystals [17], leading to $\left(\omega^{\prime}\right)^{2}=\left(\omega^{2}-k^{2} \chi\right)$. Also, the parameter of the electron-phonon coupling is renormalized to $\gamma(\mu)=\beta+\mu k \chi$, and depends on the dipole moment resulting from the transferred electron. This gives a new mechanism for the electron-phonon coupling in the charge transfer MS systems.

The total Hamiltonian is now written as

$$
H=\frac{1}{2}\left(\omega^{\prime}\right)^{2} q^{2}+H_{\mathrm{e}}(q)
$$

where the electronic part of the Hamiltonian is

$$
H_{\mathrm{e}}(q)=\delta(n)+\sigma \gamma q-t(n)
$$

The ground state energy, $W_{\mathrm{g}}(q)$, expressed as a function of intermolecular displacement is

$$
W_{\mathrm{g}}(q)=\frac{1}{2}\left(\omega^{\prime}\right)^{2} q^{2}+E_{\mathrm{g}}(q)
$$

where $E_{\mathrm{g}}(q)$ is the lowest eigenvalue of the electronic part of the Hamiltonian, $H_{\mathrm{e}}(q)$, written in the basis of the neutral and ionic states,

$$
H_{\mathrm{e}}(q)=\left|\begin{array}{ccc}
\langle N| & \left\langle I_{n}+\right| & \left\langle I_{n}-\right| \\
0 & -t(n) & -t(n) \\
-t(n) & \delta(n)+\gamma q & 0 \\
-t(n) & 0 & \delta(n)-\gamma q
\end{array}\right| .
$$

The analytical expression for the lowest eigenvalue of $H_{\mathrm{e}}(q)$ is [18]

$$
E_{\mathrm{g}}(q)=\frac{1}{3}\left[2 \delta(n)+2 G^{1 / 2} \cos \left(\phi+\frac{2}{3} \pi\right)\right],
$$

where $G=\delta^{2}(n)+3(\gamma q)^{2}+6 t^{2}(n), \phi=\frac{1}{3} \operatorname{arc} \cos \left(K G^{-3 / 2}\right)$ and $K=-\delta(n)\left[\delta^{2}(n)-\right.$ $\left.9(\gamma q)^{2}+9 t^{2}(n)\right]$. The ground state energy, $W(q)$, may show one, two or three minima, depending on the parameters, $\delta(n), \gamma$ and $\omega^{\prime}$. 


\section{Phase diagram and calculations for TTF-CA crystal}

In order to represent the variety of the ground state energy functions, $W_{\mathrm{g}}(q)$, depending on the parameters, $\delta(n), \omega^{\prime}$ and $\gamma$, I shall use a triangular diagram. At this point one should notice the similarity of the problem discussed here to that elaborated by Toyozawa [19] and represented by the T-U-S triangle (in analogy to the "alchemical triangle" introduced by Chakraverty [20]). To represent our results the triangle $\delta(n)-\omega^{\prime}-\gamma$ should be used. In molecular systems the intersite electron-electron interaction dominates the formation of ionized molecules. So the competition between electron-electron and electron-phonon interactions, represented along the U-S line on Toyozawa's [19] triangle, now is presented along the $\delta(n)-\gamma$ line. This competition leads to two extremes, the neutral MS (the single minima ground state potential) for a large enough $\delta(n) / \gamma$ ratio and the ionic dimerized stack (the two-minima potential) for a small enough $\delta(n) / \gamma$ ratio. The important observation is that one passes from a single minimum via three minima to a two minima energy curve with increasing coupling constant, $\gamma$. This is indicated schematically on the diagram (Fig. 1). It is clear from the diagram that for a soft molecular lattice (small $\omega^{\prime}$ ), and reasonably strong electron-phonon coupling (measured by the constant $\gamma$ ) that there is a region where the ground state energy function allows for the coexistence of ionic and neutral molecules. The minima of the energy function indicate stable (metastable) configurations of the MS, and values of the coordinate $q$ which correspond to the minima measure the structural changes (with respect to the neutral chain) associated with a charge density wave formed by the ionized molecules.

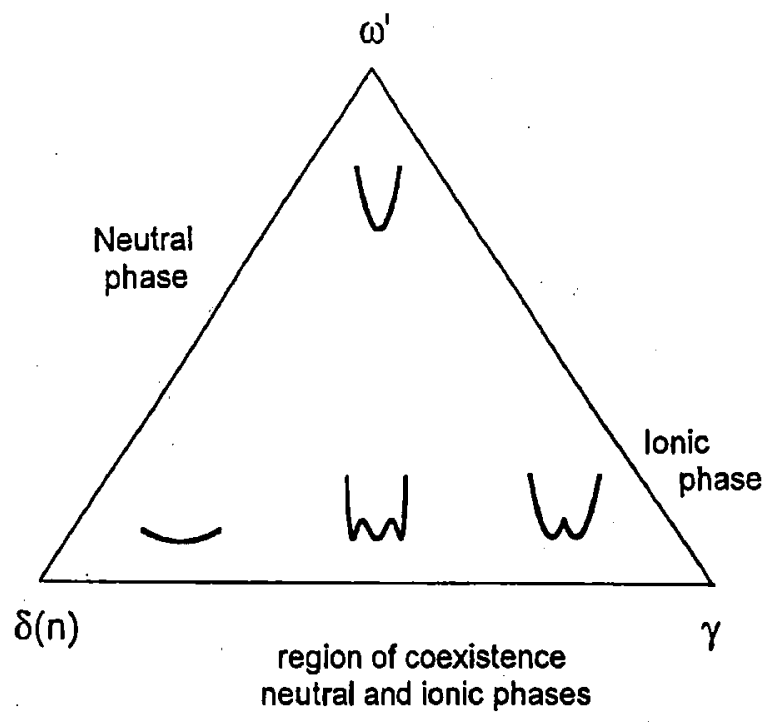

Fig. 1. A schematic ground state phase diagram for neutral and ionic phases of the mixed-stack system. 
TTF-CA a charge transfer crystal, is known to show a temperature $(80 \mathrm{~K}$ at ambient pressure), pressure (10 kbar at room temperature) and photoinduced (in low temperatures) neutral-ionic phase transition [1-3]. For this system, I adopt the following values of the parameters of the ground state energy. The Franck-Condon excitation energy for a cluster of $n$ transferred electrons in a chain is, $\delta(n)=$ $4.08-2.77 \alpha(n) \mathrm{eV}$ [21]. The transfer integral for the cluster is assumed as $t(n) \approx$ $(0.2)^{n} \mathrm{eV}$. The lattice frequency, $\omega^{\prime}$, is taken from lattice dynamics calculations [22] and for the $A_{u}, B_{u}$ modes the frequencies are in the range $35-120 \mathrm{~cm}^{-1}$. This corresponds to force constant values between 0.5 and $5 \mathrm{eV} / \AA^{2}$. The coupling constant, $\beta$, can be estimated assuming it is equal to $\alpha(n) J / a$, and results in values from $0.75(n=1)$ to $1.5 \mathrm{eV} / \AA$. The effective coupling constant, $\gamma$, is expected to be larger due to the contribution of the dipole moment of the transferred electron. So, for illustrative purposes, I assume $\gamma$ to be in the range 1.5-3.0 eV/ $\AA$. Taking $\omega^{\prime}$ equal to $100 \mathrm{~cm}^{-1}$ (force constant equal to $4 \mathrm{eV} / \AA^{2}$ ) I show in Fig. 2, the ground state energy functions calculated for different coupling constants, $\gamma$, and number of

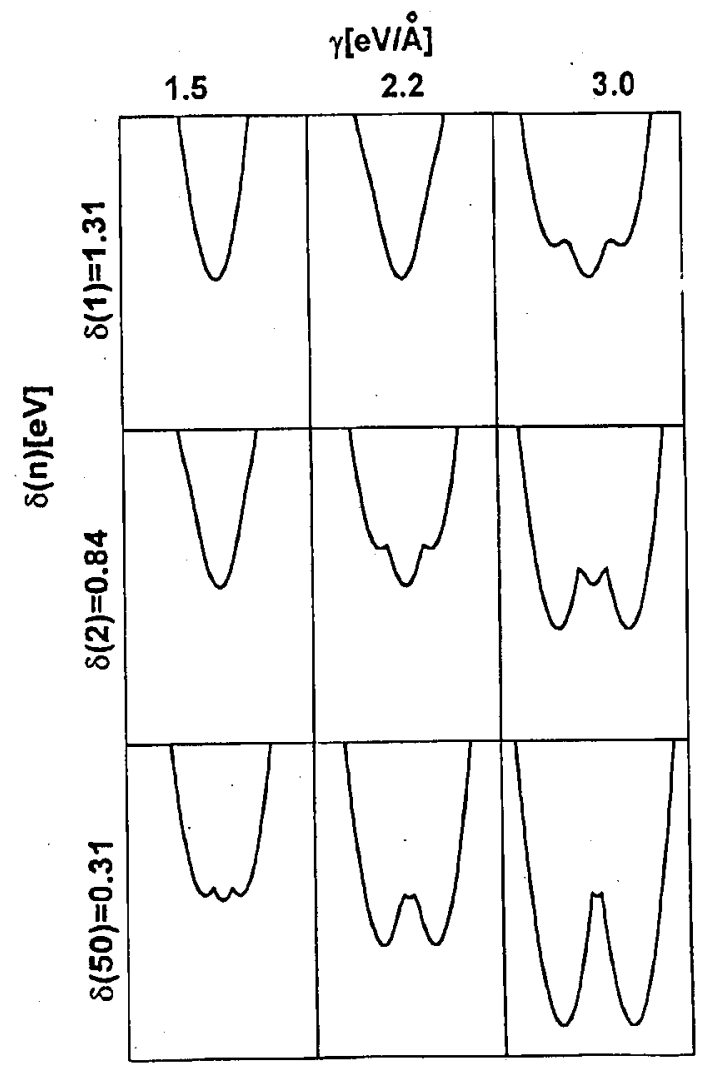

Fig. 2. Ground state energy function for TTF-CA crystal (for fixed $\omega^{\prime}=100 \mathrm{~cm}^{-1}$ ), as dependent on the parameters $\gamma$ and $\delta(n)$ (see text). 
molecules, $n$, in the cluster. Decreasing the on-site potential, $\delta(n)$, and increasing the coupling constant, $\gamma$, tends to dimerize the ionic ground state, which is what one observes tracing Fig. 2 on the diagonal.

\section{Approximation to the ground state energy function}

A common approach to approximate the ground state energy function, Eqs. (8) and (10), would be a perturbation expansion. The coupling terms, $\pm \gamma q$, on the diagonal of the electronic Hamiltonian, $H_{\mathrm{e}}(q)$, can be treated as the perturbation. This, however, has to be taken with care, since for the problem discussed, the $\gamma q$ term can be of the same order of magnitude ( $0.1 \mathrm{eV}$ for $q=0.1 \AA$ displacement) as the diagonal energy $\delta(n)(0.3-1.0 \mathrm{eV})$. On the other hand, treating the transfer integral, $t(n)$, as a perturbation would not be better. So, for an appropriate but clear insight into the dynamics of the ground state, and also for a characterization of the stable (metastable) ground states by the intermolecular coordinate, $q$, I shall expand the ground state energy in terms of the displacement coordinate, $q$. This approach is similar to that used by Toyozawa [19].

The ground state energy is approximated by Eq. (8), with

$$
E_{\mathrm{g}}(q) \approx E_{0}+a q^{2}+b q^{4}+c q^{6} \text {. }
$$

The coefficients in $E_{\mathrm{g}}(q)$ are calculated from a perturbation expansion, applied to $H_{\mathrm{e}}(q)$, Eq. (9), where the diagonal terms $\pm \gamma q$ are treated as perturbation. The unperturbed Hamiltonian is taken as

$$
H_{\mathrm{e}}(q)=\left|\begin{array}{ccc}
\langle N| & \left\langle I_{n}+\right| & \left\langle I_{n}-1\right. \\
0 & -t(n) & -t(n) \\
-t(n) & \delta(n) & 0 \\
-t(n) & 0 & \delta(n)
\end{array}\right|
$$

and diagonalized by the following states:

- the ground state, $|0\rangle=|N\rangle \cos \theta+2^{-1 / 2}\left(\left|I_{n}+\right\rangle+\left|I_{n}-\right\rangle\right) \sin \theta$, with energy

$$
E_{0}=\frac{1}{2} \delta(n)-\frac{1}{2}\left[\delta^{2}(n)+8 t^{2}(n)\right]^{1 / 2},
$$
with energy

- the first excited state (antisymmetric), $|1\rangle=2^{-1 / 2}\left(\left|I_{n}+\right\rangle-\left|I_{n}-\right\rangle>\right.$ ),

$$
E_{1}=\delta(n),
$$

- the second excited state (symmetric), $|2\rangle=2^{-1 / 2}\left(\left|I_{n}+\right\rangle+\left|I_{n}-\right\rangle\right) \cos \theta-$ $|N\rangle \sin \theta$, with energy

$$
\begin{aligned}
& E_{2}=\frac{1}{2} \delta(n)+\frac{1}{2}\left[\delta^{2}(n)+8 t^{2}(n)\right]^{1 / 2}, \\
& \tan 2 \theta=-2^{3 / 2} t(n) / \delta(n) .
\end{aligned}
$$

The electronic transitions from the ground state are

$$
\Delta E_{\mathrm{u}}(0 \Rightarrow 1)=\frac{1}{2} \delta(n)+\frac{1}{2}\left[\delta^{2}(n)+8 t^{2}(n)\right]^{1 / 2}
$$


to the lowest CT state, and

$$
\Delta E_{\mathrm{g}}(0 \Rightarrow 2)=\left[\delta^{2}(n)+8 t^{2}(n)\right]^{1 / 2}
$$

to the higher CT state. The ground state is ionized to the degree,

$$
\rho_{0}=\left(\frac{\partial E_{0}}{\partial \delta(n)}\right)=\frac{1}{2}\left\{1-\delta(n)\left[\delta^{2}(n)+8 t^{2}(n)\right]^{-1 / 2}\right\} \text {. }
$$

Here, a few remarks have to be added about the validity of our three-state model in the context of the ionicity parameter. It is a simplification of the real MS system, mostly because I do not take into account structures like $(\mathrm{DA})_{N-n}\left(\mathrm{D}^{+} \mathrm{A}^{-}\right)_{n}$, especially those which are close in energy to the $\langle N|$ and $\left\langle I_{n}+(-)\right|$ states. The contribution of the mixed states to the ground state will decay with increasing due to the decrease of their interaction with the $\langle N|$ state and increase in energy difference. Therefore, the ionicity of the ground state, defined by Eq. (13), describes the system better for smaller $(N-n)$. On the other hand, the molecular ionicity, as indirectly determined from spectroscopic experiments [23], is often understood as being defined for $N=n=1$. Nevertheless, the degree of ionicity, $\rho$, is by no means a characteristic property of MS and serves as a criterion for the neutral-ionic transition. It seems natural to express parameters of the energy function, Eq. (11), in terms of $\rho_{0}$. Another important quantity is the CT susceptibility determined by the first excited state,

$$
X_{\mathrm{u}}=2 \rho_{0} \Delta E_{\mathrm{u}}^{-1}=2 \rho_{0}\left(1-\rho_{0}\right) \Delta E_{\mathrm{g}}^{-1} .
$$

The coefficients in Eq. (11) are

$$
\begin{aligned}
& a=-X_{\mathrm{u}} \gamma^{2} \\
& b=X_{\mathrm{u}} \gamma^{4} \Delta E_{\mathrm{g}}^{-2}\left(1-3 \rho_{0}+\rho_{0}^{2}\right)\left[2\left(1-\rho_{0}\right)^{2}\right]^{-1}, \\
& c=-X_{\mathrm{u}} \gamma^{6} \Delta E_{\mathrm{g}}^{-4}\left(1-8 \rho_{0}+17 \rho_{0}^{2}-10 \rho_{0}^{3}+2 \rho_{0}^{4}\right)\left[4\left(1-\rho_{0}\right)^{4}\right]^{-1} .
\end{aligned}
$$

Now, the analysis of the ground state energy function approximated by the expansion, Eq. (11), gives the phase diagram as in Fig. 3. The diagram is presented in coordinates, $\left(E_{\mathrm{d}}, \rho_{0}\right)$, where $E_{\mathrm{d}}=2 \gamma^{2}\left(\omega^{\prime}\right)^{-2} \Delta E_{\mathrm{g}}^{-1}$, and can be interpreted as four times the reduced (divided by $\Delta E_{\mathrm{g}}$ ) deformation energy gained by the system when the electron transfer occurs. The broken line from the top of the diagram to the right lower corner is described by equation, $E_{\mathrm{d}}=-1+\rho_{0}^{-1}$, (the condition for the lattice mode softening, $\left.\left(\omega^{\prime}\right)^{2}=-2 a\right)$. Along this line, the deformation energy is equal to the energy of the lowest excited state. It describes continuous transitions up to the critical point, where the transitions become discontinuous (CB curve). The critical point is determined by the ionicity $(0.38)$ of the ground state at which the $b$ coefficient changes sign from positive to negative. The region between the CB curve and the dotted lines indicates metastability (appearance or disappearance of the side minima). In order to locate a particular system on the phase diagram, one needs to estimate the deformation energy and ionicity. For the TTF-CA crystal, an estimated value of $E_{\mathrm{d}}$ is indicated by the arrow in Fig. 3, and with the ionicity approximately 0.8 , one locates the crystal in an ionic, dimerized ground state. The ionic ground state expected for the TTF-CA crystal would explain why the neutral molecules were created by illumination of the low-temperature phase of TTF-CA [10]. One could expect that TMB-TCNQ and DMTTF-CA crystals, 


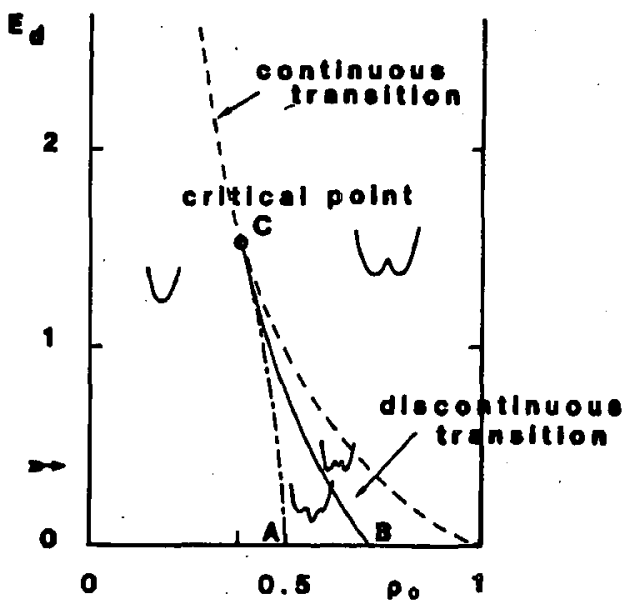

Fig. 3. Diagram $\left(E_{\mathrm{d}}, \rho_{0}\right)$ calculated with perturbative expansion of the ground state energy. The arrow indicates estimated $E_{\mathrm{d}}$ value for TTF-CA crystal.

where the pronounced coexistence of neutral and ionic molecules have been observed at low temperatures, belong to the coexistence region on the phase diagram.

The phase diagram presented in Fig. 3 is qualitatively different from the one previously proposed [13]. There are two essential differences. First, in the previous studies the electron-phonon coupling has been assumed to be off-diagonal (modulation of the transfer integral). Moreover, an equilibrium line between dimerized and undimerized phases has been defined by the condition of phonon instability. In other words, in Ref. [13], the ground state energy function (here Eq. (8)) has been approximated by a harmonic term only, while our phase diagram follows from the analysis of the energy expansion, Eq. (11). The off-diagonal and diagonal couplings influence the ground state energy differently. First of all, in a qualitative sense. This can be seen by a comparison of the harmonic coefficients in Eq. (11). Within the trimer model, $a=-2\left(1-\rho_{0}\right) \Delta E_{\mathrm{u}}^{-1} \kappa^{2}$, when off-diagonal coupling (with a constant $\kappa$ ) is assumed, while $a=-2 \rho_{0} \Delta E_{u}^{-1} \gamma^{2}$ for the diagonal coupling (constant $\gamma$ ). It follows that for a predominantly ionic ground state, the diagonal coupling is more important while off-diagonal coupling has to be taken into account for systems with a predominantly neutral ground state. This conclusion seems to confirm our intuition.

Finally, some remarks are in order about softening of the lattice mode due to the electron transfer. The perturbation expansion of the ground state energy with respect to the lattice coordinate, $q$, clearly indicates an important effect of the electron transfer on the lattice mode. One finds that the frequency of the critical lattice mode softens due to the coupling with the electron transfer and becomes, $\omega^{2}=\left(\omega^{\prime}\right)^{2}-2 X_{\mathrm{u}} \gamma^{2}$, and is strongly dependent on the degree of ionicity of the ground state. It is important to stress that $\omega^{\prime}$ is the frequency of the mode determined by all interactions, except for the electron transfer effect. This means that it is the lattice mode frequency in a neutral MS with no admixture of CT 
states. The MS architecture makes the translational displacements of molecules in opposite directions along the stack axis most difficult. This is proved by numerical calculations for the TTF-CA crystal, where such a mode has been found with the highest frequency $\left(118 \mathrm{~cm}^{-1}\right)$ for translational phonons [22]. On the other hand, the pattern of the structural change at the phase transition in the crystal indicates an important (approximately $0.1 \AA$ ) displacement of CA molecules along the stack axis [24]. This would suggest that the intermolecular electron transfer (along the stack axis) softens the $118 \mathrm{~cm}^{-1}$ phonon considerably. The estimation of the effect shows that it can be as large as $60-70 \mathrm{~cm}^{-1}$. The effect is highly anisotropic, as expected, and will be largest for phonons with polarization vectors close to the direction of the intermolecular electron transfer. More detailed, numerical calculations of the lattice dynamics for the TTF-CA crystal are planned as an extension of those previously performed [22].

\section{Conclusions}

In conclusion I would like to stress the following points. Following the suggestion from spectroscopic studies on CT excitons in MS systems, I have assumed that the modulation of the intermolecular Coulomb interaction is the essence of electron transfer-lattice vibration coupling. The interaction of the electron transfer dipole with the local electric field enhances the coupling. It modifies the on-sice potential and influences the ground state properties differently than the commonly assumed off-diagonal coupling. The important difference is that the diagonal coupling gives, in general, a three-minima ground state energy function, while the off-diagonal electron-phonon coupling alone does not allow for this property. This can be verified by diagonalization of Eq. (9) with a modulated transfer integral instead of the on-site potential. The ground state for MS systems can be viewed as resulting from the electronic interaction between diabatic states: one neutral and two ionic.

The ground state phase diagram of the MS system consists of three regions: neutral-regular stack, ionic-dimerized stack and a region, where the two phases (conpounds) can coexist. The nature of the ground state will have, obviously, consequences for the thermodynamical stability of different phases and the range of their coexistence. For the TTF-CA crystal, an estimation of the parameters of the phase diagram suggests the ionic-dimerized stack as the ground state. I suggest that TMB-TCNQ and DMTTF-CA systems are more likely to be located in the coexistence region on the phase diagram. The proposed phase diagram allows the ground state to be tuned by pressure or chemical changes. The ratio $\delta(n) / \gamma$ can be tuned, as recently shown $[4,5]$, by chemical substitution of either whole molecules or their functional groups. In many respects the charge transfer organic solids with mixed-stack architecture may successfully compete for device applications. A recently published paper [25] on bistability in molecular donor-acceptor complexes with a context of a bit storage application reports a complementary study and gives an additional value to the considerations presented here.

The analysis of the ground state energy function as performed in this paper allows to address the problem of thermodynamical stability of neutral (ionic) 
phases. This is particularly important for the TTF-CA system, where the experimental $(p-T)$ phase diagram (with the positive $\mathrm{d} p / \mathrm{d} T$ slope) has been recently well established [26]. I have suggested [27] that the diagram can be understood as follows. In the low-pressure, low-temperature region the ground state is well approximated by diabatic states and the phase space of the system is well partitioned into neutral and two ionic sub-spaces. Thus, as for a system with broken ergodicity, the corresponding components can be defined [28]. An external pressure increases quantum mixing between the diabatic states and the nature of the components changes, they become identical at a critical pressure. Temperature stimulates classical mixing of the components. Therefore, along the $p-T$ equilibrium line there is larger and larger thermal mixing of more and more similar components. The line is terminated at the critical point, where the system becomes completely homogeneous - one component in one phase. The phase transitions observed along the $p-T$ equilibrium line will become more and more continuous with increasing order-disorder character when approaching the critical point. The thermodynamics of the charge transfer organic solids will be considered in greater details elsewhere.

\section{Acknowledgment}

It is my great pleasure to dedicate this work to my Teachers and Masters, Professors Krrzysztof Pigon, Józef Rohleder and Zdzisław Ruziewicz - to commemorate their contributions to molecular solid state physics and chemistry. I appreciate the stimulation and benefit my own research and academic career have derived from their work and the warm encouragements they kindly extended to me. In particular, I thought it appropriate to present a paper on charge-transfer solids in appreciation to Professor Józef Rohleder, who introduced me to this field thirty years ago and later initiated a scientific cooperation with the Université de Rennes I.

The author wishes to acknowledge the hospitality of and discussions with Prof. Ilerve Cailleau and other colleagues from the Groupe Matiere Condensée et Materiaux, Université de Rennes I, where a part of this work has been done.

\section{References}

[1] J.B. Torrance, J.E. Vasquez, J.J. Mayerle, V.Y. Lee, Phys. Rev. Lett. 46, 253 (1981).

[2] Y. Tokura, T. Koda, T. Mitani, G. Saito, Solid State Commun. 43, 757 (1982); Y. Tokura, Y. Kaneko, H. Okamoto, S. Tanuma, T. Koda, T. Mitani, G. Saito, Mol. Cryst. Liq. Cryst. 125, 71 (1985).

[3] M.II. Lemee-Cailleau, B. Toudic, H. Cailleau, F. Mousa, M. Le Cointe, G. Silly, N. Carl, Ferroelectrics 127, 1355 (1992); J. Gallier, B. Toudic, Y. Delugeard, H. Cailleau, M. Gourdji, A. Peneau, L. Guibe, Phys. Rev. B 47, 11688 (1993) and references therein.

[4] Y. Iwasa, T. Koda, Y. Tokura, A. Kobayashi, N. Iwasawa, G. Saito, Phys. Rev. B 42, 2374 (1990); Y. Iwasawa, N. Watanabe, T. Koda, G. Saito, Phys. Rev. B 47, 2920 (1993). 
[5] S. Aoki, T. Nakayama, A. Miura, Phys. Rev. B 48, 626 (1993).

[6] see, for example, J.R. Chelikowsky, J.K. Burdett, Phys. Rev. Lett. 56, 961 (1986); A. Painelli, A. Girlando, J. Chem. Phys. 87, 1705 (1987); J.B. Torrance, J. Solid State Chem. 96, 59 (1992).

[7] A. Girlando, C. Pecile, J.B. Torrance, Solid State Commun. 54, 753 (1985).

[8] T. Luty, J. Phys. Soc. Jpn. 61, 3636 (1992).

[9] D. Haarer, M.R. Philpott, in: Spectroscopy and Excitation Dynamics of Condensed Molecular Systems, Eds. V.M. Agranovich, R.M. Hochstrasser, North-Holland, Amsterdam 1983; see also, Y.N. Gartstein, A.A. Zakhidov, P.K. Khabibullaev, Chem. Phys. Lett. 127, 263 (1986) and references therein.

[10] S. Koshihara, Y. Tokura, T. Mitani, G. Saito, T. Koda, Phys. Rev. B 42, 6853 (1990).

[11] B. Horovitz, J. Solyom, Phys. Rev. B 35, 7081 (1987).

[12] N. Nagaosa, J. Phys. Soc. Jpn. 55, 3488 (1986).

[13] A. Painelli, A. Girlando, Phys. Rev. B 37, 5748 (1988).

[14] L.Z. Stolarczyk, L. Piela, Chem. Phys. 85, 451 (1984).

[15] A. Painelli, A. Girlando, J. Chem. Phys. 84, 5655 (1986), ibid. 87, 1705 (1987).

[16] T. Lu'y, J. Chem. Phys. 87, 3137 (1987).

[17] T. Luty, J. Chem. Phys. 66, 1231 (1977); R.W. Munn, Mol. Phys. 64, 1 (1988).

[18] A.G. Every, Phys. Rev. B 22, 1746 (1980).

[19] Y. Toyozawa, J. Phys. Soc. Jpn. 50, 1861 (1981); K. Nasu, Y. Toyozawa, ibid. 51, 2098, 3111 (1982).

[20] B.K. Chakraverty, Nature 287, 393 (1980).

[21] R.M. Metzger, J.B. Torrance, J. Am. Chem. Soc. 107, 117 (1985).

[22] P. Levy, H. Bonadeo, Chem. Phys. Lett. 144, 377 (1990); T. Luty, K. Rohleder, Mol. Cryst. Liq. Cryst. 229, 141 (1993).

[23] A. Girlando, R. Bozio, C. Pecile, J.B. Torrance, Phys. Rev. B 26, 2306 (1982); C.S. Jacobsen, J.B. Torrance, J. Chem. Phys. 78, 112 (1983).

[24] M. Le Cointe, M.H. Lemee-Cailleau, L. Toupet, B. Toudic, H. Cailleau, G. Heger, F. Moussa, in: Proceedings of Disorder in Molecular Solids (III) Conference, Garchy (France), 1993.

[25] N. Guihery, G. Durand, M.B. Lepetit, Chem. Phys. 183, 45 (1994).

[26] M. Le Cointe, Ph.D. Thesis, Université de Rennes I, 1994.

[27] T. Luty, Fifth French-Polish Seminar on Phase Transformations and Dynamics of Molecular Materials, Saint-Malo (France), 1994, unpublished.

[28] R.G. Palmer, Adv. Phys. 31, 669 (1982). 